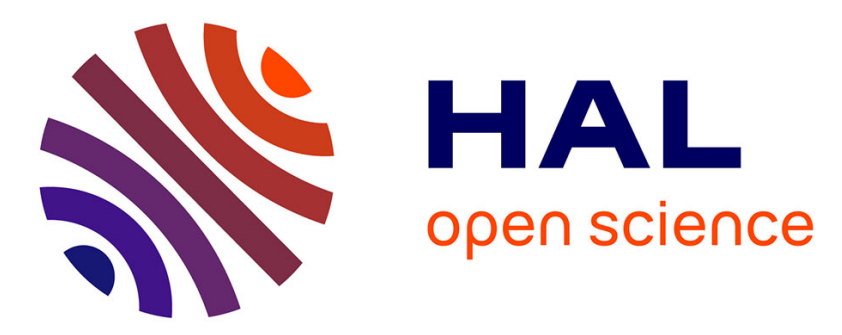

\title{
Défaite militaire et révolution antidémocratique. Le parallèle entre l'Athènes de 404 et la France de 1940 dans Les Oligarques de Jules Isaac.
}

Dominique Lenfant

\section{- To cite this version:}

Dominique Lenfant. Défaite militaire et révolution antidémocratique. Le parallèle entre l'Athènes de 404 et la France de 1940 dans Les Oligarques de Jules Isaac.. KTÈMA Civilisations de l'Orient, de la Grèce et de Rome antiques, 2017, 42, pp.183-194. halshs-01669243

\section{HAL Id: halshs-01669243 \\ https://shs.hal.science/halshs-01669243}

Submitted on 21 Dec 2017

HAL is a multi-disciplinary open access archive for the deposit and dissemination of scientific research documents, whether they are published or not. The documents may come from teaching and research institutions in France or abroad, or from public or private research centers.
L'archive ouverte pluridisciplinaire HAL, est destinée au dépôt et à la diffusion de documents scientifiques de niveau recherche, publiés ou non, émanant des établissements d'enseignement et de recherche français ou étrangers, des laboratoires publics ou privés. 

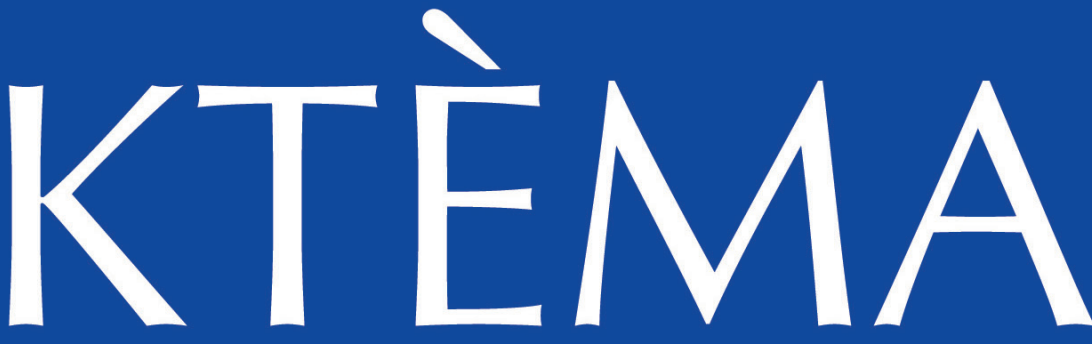

\section{CIVILISATIONS DE L'ORIENT, DE LA GRÈCE ET DE ROME ANTIQUES}

Edith Foster

Edmond LÉvY

Tim Roop

Cinzia Bearzot

\section{Michel Нumm}

David S. Levene

Estelle OudoT

Hans Kopp

Maciej JUNKIERT

Tobias Јоно

Christian WENDT

Oliver SCHELSKe

Dominique Lenfant

Neville MORLEY

Agnès Molinier Arbo

Michèle Coltelloni-Trannoy Philippe Torrens

Jean Ducat

Michel WoronofF

\section{Les interprétations de la défaite de 404}

Interpretations of Athen's defeat in the Peloponnesian war............................................................ 7

Thucydide, le premier interprète d'une défaite anormale .............................................................. 9

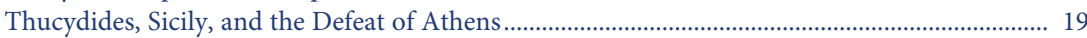

La $\sigma u \mu \varphi$ ód de la cité

La défaite d'Athènes (405-404 av. J.-C.) chez les orateurs attiques.............................................. 41

Rome, une «cité grecque» prise par les Hyperboréens.................................................................... 53

Rome Redeems Athens?

Livy, the Peloponnesian War, and the Conquest of Greece............................................................ 73

Ultime défaite d'Athènes ou sa plus belle victoire?

Stratégies rhétoriques autour de la bataille d'Aigos-Potamoi

dans le Panathénaïque d'Aelius Aristide

The Defeat of Athens in 404 BC in The Federalist ............................................................................ 97

Polish Reflections: The Reception of the Defeat of Athens in

the Works of Gottfried Ernst Groddeck and Joachim Lelewel.........................................................115

The Internal Commotion of Greek Culture: Jacob Burckhardt

on the Defeat of Athens in the Peloponnesian War.

Spree-Athen nach dem Untergang

Eduard Meyer zur Parallelität von Geschichte

Der Kampf um die Demokratie

Thukydides in Deutschland nach dem Ersten Weltkrieg

Défaite militaire et révolution antidémocratique

Le parallèle entre l'Athènes de 404 et la France de 1940

dans Les Oligarques de Jules Isaac.

Thucydides and the Historiography of Trauma.

\section{Le thauma dans l'historiographie grecque d'époque impériale}

Hérodien, Rome et le spectacle du pouvoir

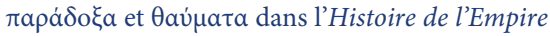

après la mort de Marc Aurèle

La place du thauma dans l'Histoire romaine de Cassius Dion............................................................219

Le lexique de l'étonnement chez Appien. Quelques remarques .......................................................23

\section{Varia}

Du caractère «mixte» du régime spartiate 251

L’image de la défaite dans l'Iliade. 


\author{
KTÈMA \\ CIVILISATIONS DE L'ORIENT, DE LA GRÈCE ET DE ROME ANTIQUES \\ Revue annuelle \\ Fondateurs : Edmond Frézouls † \\ Edmond LÉvY
}

Comité Directeur: Dominique Beyer, Bruno Bleckmann, Jean-François Bommelaer, Frédéric Colin, MireilleCorbier, GérardFreyburger, Jean Gascou, Jean-Georges Heintz, Michel Humbert, Anne Jacquemin, Stavros Lazaris, Dominique Lenfant, Edmond Lévy, Jean-Claude Margueron, Henriette Pavis d'Escurac, Laurent Pernot, Thierry Petit, Gérard SiEBERT

Rédaction:Edmond LÉvy

Dominique Beyer et Gérard Freyburger

Maquette et mise en page: Ersie LERIA

\title{
Éditeur
}

Presses universitaires de Strasbourg

5 allée du Général Rouvillois - CS50008

FR-67083 Strasbourg Cedex

Tél: (33) 0368856265

info.pus@unistra.fr

pus.unistra.fr

\section{Ventes au numéro}

En librairie ou en commande en ligne sur le site du Comptoir des presses d'universités: www.lcdpu.fr

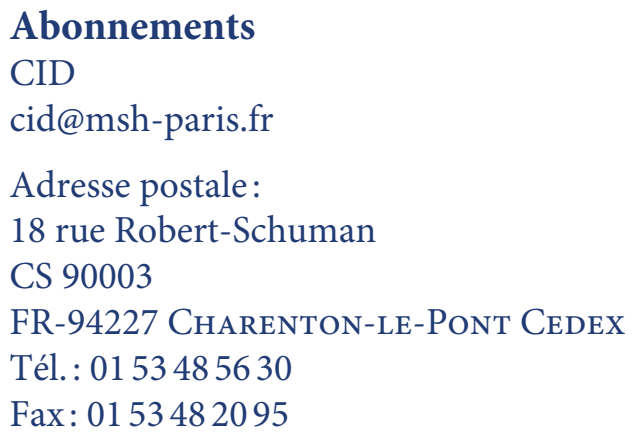




\section{Défaite militaire et révolution antidémocratique Le parallèle entre l'Athènes de 404 et la France de 1940 dans Les Oligarques de Jules Isaac}

Salut public, que de crimes on commet en ton nom!

Jules Isaac ${ }^{1}$

RÉsumé-. En 1942, l'historien Jules Isaac achève dans la clandestinité un ouvrage intitulé Les Oligarques. Il y traite des adversaires athéniens de la démocratie qui, en 404 avant J.-C., profitèrent de la défaite face à Sparte pour renverser le régime avec l'assistance de l'ennemi. Le sujet lui est inspiré par ses ressemblances avec l'actualité, après la défaite de la France face aux Allemands, la mise en place d'un gouvernement antidémocratique collaborant avec Hitler, l'abolition générale des libertés, puis les persécutions contre les Juifs, à commencer par la dégradation civique de Français tels que l'auteur. En retour, cette actualité influence l'interprétation que ce dernier propose des événements antiques, dans un ouvrage au genre délibérément inclassable.

Aвstract-. Jules Isaac wrote Les Oligarques in hiding in 1942. This book focusses on Athenian adversaries of democracy who took advantage of Athens' defeat by Sparta in order to dissolve the democracy with the help of the enemy. Resemblances to contemporary events, once France was defeated by Germany, inspired Isaac to develop this subject: the installation of an anti-democratic government that collaborated with Hitler, the general abolition of civil liberties, the persecutions of Jews, in particular the civic degradation of French citizens such as the author himself, influenced Isaac's interpretation of the ancient events in a book that deliberately defies classification according to genre.

(1) Jules IsaAC, Les Oligarques, Paris, 1989, p. 69 (dans la réédition par Calmann-Lévy en 1989, à laquelle renverront toutes nos indications de pages, cette édition étant la plus largement accessible), à propos (entre autres) de Pisandre, qui, en 411 av. J.-C., invoqua le salut public pour renverser la démocratie (Thucydide, VIII, 53,2). L'exclamation est calquée sur celle qu'aurait eue Madame Roland avant d'être guillotinée par les Révolutionnaires en 1793, "Ô Liberté, que de crimes on commet en ton nom!».

Les Oligarques ont été composés entre 1941 et octobre 1942, publiés une première fois en avril 1945 aux éditions de Minuit (Junius, Les Oligarques. Essai d'histoire partiale, Paris, Éditions de Minuit, 1945) et une deuxième fois en 1989 chez Calmann-Lévy, avec une préface de Pascal Ory (Jules IsAac, Les Oligarques. Essai d'histoire partiale, Paris, CalmannLévy, 1989). Ce n'est que tout récemment, en 2016, qu'ils ont fait l'objet d'une première traduction, en italien, par Paolo Fai, précédée d'une introduction de Luciano Canfora (Jules IsAAC, Gli oligarchi: saggio di storia parziale, introduzione di Luciano Canfora, traduzione di Paolo Fai, Palerme, 2016).

Le livre a donné lieu à des comptes rendus des antiquisants André Aymard (Revue des Études Anciennes 49/3-4, 1947, p. 321-326) et Paul Cloché (Revue Historique 200, 1948, p. 109-111) et, cinquante ans plus tard, à deux articles d'analyse parus dans des volumes collectifs peu diffusés: Olivier CURTy, "Quelques observations sur Les Oligarques de Jules Isaac», dans I. Lewandowski et L. Mrozewicz (éd.), L’image de l'Antiquité chez les auteurs postérieurs, Poznań, 1996, p. 171-182, et Christophe Chandezon, «Les Oligarques: une histoire comparée», dans Les engagements de Jules Isaac. Actes du colloque d'Aix-en-Provence (27 et 28 mars 1997), Aix-en-Provence, 1998, p. 97-106. 
Les Oligarques sont un ouvrage doublement lié à la défaite, par son sujet et par le contexte de sa rédaction. C'est, en effet, après la défaite de la France face aux Allemands en juin 1940 qu'il fut composé dans la clandestinité: commencé en 1941, il fut achevé en octobre 1942, dans la France occupée par l'Allemagne nazie ${ }^{2}$. Ce contexte est déterminant, puisque le choix du sujet (les oligarques athéniens) est explicitement dû à la perception d'une forte ressemblance entre l'expérience contemporaine et un épisode de l'histoire antique, à plus de vingt-trois siècles de distance ${ }^{3}$.

Cette expérience commune est celle d'un pays qui a subi une défaite militaire face à l'ennemi (la France face à l'Allemagne, comme Athènes face à Sparte) et où cette défaite, sanctionnée par un accord politique, a été rapidement suivie de l'abolition du régime démocratique (dans la France de 1940, l'État français ou régime de Vichy remplace la Troisième République, comme à Athènes, le régime des Trente succède à la démocratie en $404 \mathrm{av}$. J.-C.), une abolition imputable à des citoyens qui étaient à la fois des adversaires de la démocratie et des collaborateurs de l'ennemi vainqueur de leur patrie.

C'est au rôle de ces adversaires de la démocratie athénienne qu'est consacré l'ouvrage, à ceux que les Grecs appelaient les oligarques ${ }^{4}$. Jules Isaac ne se soucie pas tant de la défaite en $\operatorname{soi}^{5}$ que de son exploitation par les ennemis de la démocratie. Cet angle d'approche particulier a toute sa place dans l'histoire des interprétations de la défaite de 404 , à laquelle est consacré le présent dossier d'articles $^{6}$, mais il importe de l'analyser dans toute sa singularité, tant celle-ci a de quoi dérouter.

\section{CLASSICISME ET SINGULARITÉS}

Sur le plan formel, il s'agit d'un livre de 200 pages qui a l'allure d'une étude historique ${ }^{7}$. Il est de facture classique, avec un premier chapitre qui présente les acteurs qui sont l'objet déclaré de l'étude (les oligarques athéniens), puis cinq autres chapitres, qui suivent un plan sagement chronologique et traitent successivement de l'affaire des hermès de 415 (ch. II), de la Révolution des Quatre Cents (ch.III), du désastre de 405 et de l'entrée en scène des oligarques (ch.IV), de l'exploitation de ce désastre par les Trente Tyrans (ch.V) et enfin du processus de retour à la démocratie grâce au combat des démocrates de Thrasybule et à l'action du roi de Sparte Pausanias (ch. VI). Le livre a donc pour objet les oligarques athéniens dans une période très restreinte, d'une quinzaine d'années, de 415 à 401 .

L'auteur se fonde en grande partie sur les sources littéraires grecques: Thucydide, puis Xénophon et Lysias, parfois aussi sur la Constitution des Athéniens aristotélicienne, le pamphlet homonyme

(2) L'auteur date lui-même les «ultimes lignes» de son livre du 17 octobre 1942. La France disposait encore d'une zone sud dite «libre», puisque ce fut le mois suivant, en novembre 1942, que les Allemands et les Italiens occupèrent également celle-ci. C'est à Aix-en-Provence, en zone libre, que l'auteur composa cet ouvrage. Ce dernier ne fut publié qu'à la Libération, en avril 1945.

(3) 2346 ans de distance, précise l'auteur lui-même au seuil de son ouvrage, p. 8.

(4) L'auteur l'indique dès ses premiers mots: «Je me propose de montrer quel fut le rôle du parti oligarchique d'Athènes, ennemi mortel de la démocratie, dans les événements qui précédèrent, déterminèrent et suivirent la chute désastreuse de la cité, à la fin de la guerre du Péloponnèse» (p. 8).

(5) À l'inverse, Marc BLoch essaie dès 1940 d'analyser les causes de la défaite française dans ce qui paraîtra après sa mort sous le titre de L'Étrange défaite.

(6) La présente contribution a fait l'objet d'une communication lors du colloque Defeat and the West: Interpretations of the Defeat of Athens in 404 BCE organisé à Strasbourg les 13 et 14 mai 2016 par Edith Foster, alors membre de l'Institut d'études avancées de Strasbourg, en collaboration avec l'UMR 7044 Archimède. Je remercie les participants du workshop pour tous leurs commentaires.

(7) 201 pages dans l'édition originale de 1945, 180 pages dans la réédition de 1989. 
du Pseudo-Xénophon (la Constitution des Athéniens), Andocide, Diodore ou Plutarque. Il renvoie ici et là à tel et tel passage dans les notes de bas de page, quoique de manière moins systématique qu'on ne l'attendrait d'un ouvrage spécialisé. Selon ces critères formels (structure classique, objet restreint, renvoi limité aux sources), Les Oligarques ont l'allure d'un livre d'histoire destiné à un public cultivé.

Ces dehors classiques cachent néanmoins plusieurs originalités:

1. Le récit est traversé de bout en bout par une thèse précise.

2. Il repose sur l'établissement d'analogies entre le passé et le présent.

3. Il est délibérément imprégné de jugements de valeur pleinement revendiqués.

\section{Un récit à thèse}

Une thèse traverse l'ouvrage, dans laquelle la défaite a sa place. Elle pose, d'abord, qu'il y avait à Athènes, dès avant sa défaite face à Sparte, des ennemis de la démocratie et de la patrie, des «ennemis du dedans, mille fois plus implacables que ceux du dehors» (p. 26), ensuite que ces hommes se sont réjouis de la défaite de leur cité parce qu'elle leur a donné l'occasion de prendre le pouvoir avec l'aide de l'ennemi. Plus même: ils avaient appelé «de leurs vœux le désastre libérateur» ${ }^{8}$.

C'est une thèse ou une hypothèse que l'on peut légitimement qualifier d'historique (ce qui n'empêche pas de la contester). De 415 à 404, l'auteur suppose ces oligarques aux aguets, se réjouissant des malheurs de leur patrie, voire y contribuant dans la coulisse. Ainsi, en 415, les auteurs des sacrilèges sont présentés comme des oligarques ayant voulu renverser la démocratie (p. 48-52). Puis, le désastre subi en Sicile en 413 est, selon l'auteur, le but même qu'ils s'étaient proposé (p. 53-54) et pour eux une raison de se réjouir, du fait qu’il a "profondément ébranlé» le régime:

Pour des conspirateurs aux aguets, ennemis jurés de la démocratie, le désastre n'ouvrait-il pas des perspectives réjouissantes? «Divine surprise», le régime en était profondément ébranlé; discrédités, les habituels conseillers du peuple $([\ldots])$; l'opinion, déchaînée et démontée, semblait prête à tout accepter. (p. 58)

Le Conseil de Dix institué dans la foulée est, d'après l'auteur, dû à leur influence, et s'il est sans conséquence immédiate, c'est qu'ils ne sont pas prêts à l'action. Néanmoins, les hétairies ou sociétés secrètes oligarchiques font ensuite, dit-il, un effort de recrutement et d'organisation en vue de renverser la démocratie (p.60). Les oligarques prennent le pouvoir en 411, mais c'est finalement une «Révolution manquée», parce qu'elle n'obtient pas l'appui de l'ennemi et rencontre la résistance des Athéniens à Samos (ch. III). Le déroulement du procès des Arginuses est imputé par l'auteur à l'action des oligarques (ch. IV). Puis il affirme qu'après le désastre militaire d'Aigos Potamoi, c'est pour les oligarques un «jeu d'enfant que de discréditer la démocratie et ses chefs en rejetant sur eux toute la responsabilité du désastre» (p. 107). La capitulation de 404 est pour les oligarques une source de jouissance et d'espérance (p. 113). Ces derniers collaborent avec l'ennemi pour imposer au peuple le régime de leurs vœux (ch.V). Ils se livrent à tous les crimes, règnent dans la terreur et, pendant la guerre civile, n'ont d'autre souci que d'asseoir leurs propres privilèges (p. 179). Une fois la démocratie restaurée, ils bénéficient de la clémence des démocrates (ch.VI).

Selon cette optique, les oligarques formeraient un groupe agissant ou conspirant de manière constante durant toutes ces années, au cours desquelles ils seraient montés en puissance jusqu'à obtenir le pouvoir grâce à la défaite et à l'ennemi. Cette thèse peut à première vue sembler originale par le poids majeur qu'elle accorde à ces acteurs. Elle peut aussi paraitre inspirée de l'actualité de l'époque: la présence en France d'une extrême droite très active dès avant l'arrivée des nazis. Cela

(8) p. 29. Cf. p. 19: «tout désastre vous sera triomphe s'il précipite la ruine de la démocratie abhorrée.» 
nous conduit à parler de la deuxième originalité de cet écrit, le fait qu'il repose sur l'établissement d'analogies entre le passé et le présent.

\section{Le rôle des analogies entre le passé et le présent}

Ces analogies ne sont pas signalées de bout en bout, mais elles sont clairement suggérées par trois procédés.

Il y a d'abord les formules qui encadrent l'ensemble. Dans l'avertissement initial (p. 8), l'auteur juxtapose de manière significative la mention des deux défaites, celle d'Athènes et celle de la France à propos de laquelle il écrit:

C'est en 404 avant J.-C. qu'Athènes vaincue dut plier les genoux devant Sparte. C'est en 1942 après

J.-C., dans la France subjuguée par l'Allemagne hitlérienne, que ces pages ont été écrites.

Quant à la formule finale (p. 191), elle signale que les oligarques sont toujours aussi malfaisants et l'auteur se demande si les démocrates seront aussi magnanimes que leurs équivalents antiques ${ }^{9}$ : c'est le seul passage qui compare explicitement les deux situations ${ }^{10}$.

Il y a ensuite le recours, pour décrire les réalités grecques, à des formules empruntées à l'extrême droite française et signalées par des guillemets. Ainsi, l'idée même que le désastre militaire permette aux oligarques d'espérer une révolution est clairement inspirée de l'analogie avec juin 1940: à propos du désastre de 413, l'auteur parle de "divine surprise», expression par laquelle Charles Maurras (l'idéologue d'extrême droite dont les idées ont inspiré Vichy) avait qualifié la défaite face à l'Allemagne ${ }^{11}$. De même, à propos des lendemains de la défaite de 404, il écrit que les oligarques «ne pouvaient concevoir le proche avenir d'Athènes sans la rénovation oligarchique (Athènes aux Athéniens, aux Athéniens «réels») et cette rénovation elle-même sans le concours de l'étranger, de l'ennemi, du vainqueur (Athènes aux Spartiates), puisque aussi bien il fallait l'imposer de force à un peuple visiblement réfractaire.» (p. 123). L'historien transfère délibérément dans l'Athènes antique des expressions des partisans de l'extrême droite antisémite française: "Athènes aux Athéniens", par exemple, fait écho à la devise d'extrême droite «la France aux Français » ${ }^{12}$.

Il y a enfin l'énoncé de généralités sur les comportements politiques, qui sont données pour valables de tout temps - et qui s'appliquent tout particulièrement aux années 1940. Par exemple, à propos du conseil de dix Anciens qui fut institué à la suite du désastre de Sicile (ils étaient chargés de donner un avis préalable sur les mesures à prendre) ${ }^{13}$, l'auteur signale la participation de Sophocle, alors plus qu'octogénaire ${ }^{14}$, et commente: «le nom vénéré d'un illustre vieillard fait bien sur la façade de certaines entreprises de démolition; il aide à camoufler les démarches les plus

(9) «[... ] les «bons» sont toujours aussi malfaisants; savoir si les «méchants» seront aussi magnanimes.». Isaac emploie la terminologie des oligarques antiques et en dénonce l'inanité par ses guillemets (les oligarques se qualifiaient volontiers de «bons» - eux et, plus largement, l'élite - et désignaient le peuple des démocrates comme étant les «méchants» (ainsi,

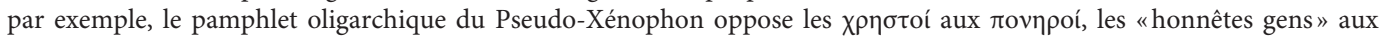
«fripons»).

(10) Chandezon (supra n. 1, p. 97) souligne à juste titre que Les Oligarques sont un «livre d'espoir», puisque le régime des Trente a débouché sur la restauration de la démocratie. C'est effectivement une analogie espérée par l'auteur, qui ne doute pas que la sombre période en cours ne s'achève. Il est d'autant plus frappant de voir qu'il n'évoque l'avenir que pour s'inquiéter du futur sort des collaborateurs et de l'attitude des démocrates à leur encontre.

(11) L'expression est reprise avec insistance: p. 54, 55, 58.

(12) «La France aux Français» était le sous-titre du journal d'extrême droite antisémite La libre parole et la devise de la Fédération nationale antijuive fondée en 1903 par Édouard Drumont. De même, les termes de «rénovation» et de «réfractaire» relèvent de la propagande vichyste. Le réfractaire désignait alors le résistant qui refusait de partir travailler en Allemagne. Parmi les autres expressions ouvertement empruntées à l'extrême droite française et signalées par des guillemets figurent encore «loyal échange de services», «collaboration confiante» et «l'ordre moral» (p. 143).

(13) Thucydide, VIII, 1, 3 (cf. Aristote, Constitution des Athéniens, 29, 2).

(14) Cf. Aristote, Rhétorique, 1419a 25. 
suspectes: cela aussi est de tous les temps.» - une allusion évidente à Pétain, 84 ans en $1940^{15}$. De même, quand il rend compte de la défiance mutuelle qui s'instaure entre les citoyens athéniens à la suite des assassinats politiques et de la terreur que sèment les oligarques avant de s'emparer du pouvoir en 411, Isaac commente: «Sous de tels orages, la réaction humaine est partout la même: une floraison instantanée, une splendide floraison de lâcheté» $(\text { p. } 72)^{16}$.

Ce ne sont là que les indices les plus transparents. L'analogie est partout implicite, sans que soit clairement évoqué le pendant du $\mathrm{xx}^{\mathrm{e}}$ siècle. Ainsi, à propos des attaques subies par les métèques «en dépit des loyaux services que la plupart avaient rendus à leur cité d'adoption» et dans lesquelles «la xénophobie», la «trahison», la «cupidité», la «doctrine» jouent leur rôle chez des oligarques qui spéculent "sur des sentiments de basse jalousie» (p. 145), on songe inévitablement aux persécutions contre les Juifs dans la France des années $1940^{17}$.

\section{Une partialité affichée}

Au-delà de sa thèse et des analogies entre époques, la troisième originalité du livre est sa partialité affichée: par l'abondance de ses jugements de valeur, son recours à l'ironie ou aux qualificatifs moraux, l'auteur a bien souvent le ton d'un polémiste.

C'est ainsi que le chapitre V s'intitule "L'exploitation scélérate du désastre», qu'Antiphon est qualifié de «doctrinaire ranci» (p.62) ou que l'auteur ironise sur la prétendue «régénération» voulue par les oligarques:

Telle était la vie quotidienne des Athéniens sous le gouvernement des Trente: citoyens ou métèques, chacun pouvait se dire menacé; la sécurité quotidienne avait disparu, avec le respect des lois. Et tel était le régime: chasse à l'argent, chasse à l'homme, violence, pillage et meurtre. On appréciera ces méthodes de «régénération». (p. 148)

Ou encore, à propos des oligarques qui, dans la guerre civile athénienne, refusèrent dépargner leur patrie comme l'en suppliaient leurs adversaires:

Mais la patrie, qu'était-ce pour ces fanatiques: leur caste, les Athéniens bien nés, bien pensants, bien pourvus, le "pays réel», eux-mêmes, leurs intérêts, leurs biens, leurs privilèges, pour la sauvegarde desquels ils étaient prêts à payer n'importe quel prix, à s'accommoder de n'importe quelle servitude.

Le Démos enchaîné, le Spartiate à l'Acropole, telle était leur formule patriotique. (p. 179)

Une telle diatribe paraît inimaginable dans un récit d'historien ou dans un contexte où le référent serait vieux de vingt-trois siècles. L'objet de l'étude est clairement désigné comme le mal, tandis qu'à l'inverse l'auteur dit sans fard son amour pour la démocratie et la liberté qu'elle garantit (p. 12-17). Même admiration explicite pour la clémence dont firent preuve les démocrates athéniens au moment de la réconciliation civique de 403 - et, nous l'avons vu, les derniers mots de l'ouvrage sont pour se demander si leurs équivalents contemporains seront aussi «magnanimes».

Ces caractéristiques peuvent surprendre au vu des règles que l'historiographie moderne cherchait à promouvoir depuis déjà quelque temps, mais aussi eu égard à la personnalité de

(15) p. 58-59. Autre allusion à Pétain, à propos de la capitulation de 404: «Athènes plaçait tout son espoir dans le génie cauteleux de celui en qui elle ne voulait plus voir que l'homme de la paix» (p. 113).

(16) Signalons aussi les portraits d'oligarques ou de démocrates qui peuvent être vus comme les analogues d'hommes politiques français, notamment p. 61-71. Sur de possibles correspondants, cf. Pascal ORY, «Préface», dans Jules Isaac, Les Oligarques. Essai d'histoire partiale, Paris, Calmann-Lévy, 1989, p. X (Périclès et Léon Blum, Antiphon et Charles Maurras, Pisandre et Jacques Doriot); André Kaspi, Jules Isaac ou La passion de la vérité, Paris, Plon, 2002, p. 157-158 (Thrasybule et de Gaulle $[\ldots])$.

(17) Le parallèle français est tellement absent qu'on ne peut affirmer avec certitude que certaines analogies aient été réellement présentes dans l'esprit de l'auteur. Chandezon (supra n. 1, p. 103) se demande, par exemple, si les hétairies étaient censées rappeler les ligues de l'entre-deux-guerres. 
l'auteur, auquel nous arrivons enfin - après avoir intentionnellement différé ce moment (l'ouvrage n'a jamais paru de son vivant que sous un pseudonyme ${ }^{18}$ ).

\section{UN AUTEUR HORS NORMES}

On se contentera de souligner deux points à ce stade de sa vie. Tout d'abord, Jules Isaac n'est pas un anonyme ni un simple amateur. Il sait ce qu'est l'histoire, puisqu'il a été professeur d'histoire au lycée pendant près de trente-cinq ans avant de contribuer à partir de 1936, en tant qu'inspecteur général de l'instruction publique, à l'élaboration des programmes scolaires et à la formation des professeurs d'histoire. Il est en fait, depuis des décennies, un pilier de l'enseignement de l'histoire en France. Son nom est connu de nombre d'élèves et anciens élèves français, car il est l'auteur de manuels scolaires qui ont eu cours dans l'enseignement secondaire français dans les vingt années qui précèdent la Seconde Guerre mondiale, le fameux «Malet-Isaac» - le manuel qui a rencontré le plus grand succès auprès des professeurs d'histoire des années trente ${ }^{19}$. Jules Isaac est aussi un humaniste et il a milité entre les deux guerres pour la paix et pour l'entente franco-allemande ${ }^{20}$.

En second lieu, il a 65 ans quand il achève Les Oligarques. Il fait partie d'une famille juive installée en France depuis des générations et régulièrement active dans le service public: avant ses propres services dans l'enseignement, son père et son grand-père avaient fait carrière dans l'armée; et quand l'Alsace est devenue allemande en 1871, son père a choisi, comme beaucoup d'autres Français, de migrer vers l'intérieur de la France pour pouvoir rester français ${ }^{21}$. Or, en octobre 1940, le gouvernement de Vichy prend les premières mesures discriminant les Juifs, avec le Statut des Juifs qui interdit désormais aux Juifs français d'être fonctionnaires ou professeurs. Jules Isaac est alors révoqué. Il commence par se réfugier en zone libre (en Provence) ${ }^{22}$ et c'est dans ces circonstances qu'il compose Les Oligarques, en 1941-1942.

L'auteur n'est donc pas un spécialiste de l'Antiquitée ${ }^{23}$, ce n'est pas non plus un chercheur, mais c'est un pédagogue qui sait pratiquer la synthèse et la rigueur, et c'est un généraliste, qui connaît ses classiques ${ }^{24}$ et qui a composé des manuels sur toutes les périodes, de l'Antiquité à l'époque contemporaine ${ }^{25}$. Notons que le «Malet-Isaac» de 1930 destiné aux élèves de $6^{\mathrm{e}}$ (11 ans) consacre plus de 400 pages à l'Orient et à la Grèce antiques.

(18) Voir infra, III. 3.

(19) Cf. Kaspi (supra n. 16), p. 104. Le Malet-Isaac a eu cours jusque dans les années 1970, soit durant près d'un demisiècle. Albert Malet, de 13 ans l'aîné d'Isaac, avait inauguré la collection, mais était mort en 1915 lors de la Première Guerre mondiale. Isaac, qui avait d'abord collaboré avec Malet, fut chargé de réviser et d'actualiser ces manuels et d'en poursuivre la rédaction, avant d'en composer de nouveaux pour s'adapter aux programmes scolaires successifs. Sur ces manuels et leurs grandes caractéristiques, cf. Alice GérARD, "Origines et caractéristiques du Malet-Isaac », dans Jules Isaac. Actes du colloque. Catalogue de l'exposition organisée par l'Université de Haute-Bretagne (Rennes II), 28 novembre-10 décembre 1977, Paris, Hachette, 1979, p. 52-59; Charles Olivier CARbonelL, «L'originalité de la collection «Jules Isaac» », dans Jules Isaac. Actes $d u$ colloque..., 1979, p. 68-80; KASPI (supra n. 16), p. 73-113.

(20) Cf. Kaspi (supra n. 16), p. 114-137.

(21) Cf. Kaspi (supra n. 16), p. 14-15 et 18.

(22) Il se cachera ensuite au Chambon-sur-Lignon, puis à Riom, où sa femme sera arrêtée par la Gestapo en octobre 1943, peu après son fils cadet, sa fille et son gendre. Ils seront déportés à Auschwitz (son gendre à Buchenwald), et seul son fils en réchappera. Cf. KASPI (supra n. 16), p. 160-161 et 166-168.

(23) Cela le distingue d'un Eduard Meyer, dont traite Christian Wendt dans ce même numéro de Ktèma.

(24) Chandezon (supra n. 1, p. 98) rappelle opportunément que, dans un lycée classique de la fin du Xix siècle, les élèves apprenaient les langues anciennes et avaient avec les «grands textes» une familiarité qui n'est plus d'actualité.

(25) KASPI (supra n. 16), p. 149. 
Le choix de l'histoire grecque pourrait surprendre, dans l'absolu. Mais, dans ces circonstances, l'auteur le justifie, au seuil de son livre, par la distance, et le présente comme un moyen de «s'enfuir dans le temps ${ }^{26}$. Cette fuite n'est que de façade, puisque ce sont précisément les ressemblances de situation et de comportement qui le font remonter de l'actualité française à l'Athènes antique - les deux défaites qui ont fait le lit d'oligarques aux aguets. Ajoutons qu'Isaac est un admirateur de la démocratie athénienne, dont il ne manque pas d'exalter le principe de liberté, tout comme il est attaché à leur équivalent dans la France moderne: dans sa dédicace, il présente son livre comme un «hymne à la divine liberté perdue» et, plus de vingt ans après, il se souvient que, «face au régime hypocrite issu de la défaite», son "premier mouvement fut d'affirmer, de crier [son] amour fervent des libertés démocratiques ${ }^{27}$.

Le contexte, c'est aussi le choc que vient de vivre Jules Isaac dans ces années 1940, non pas tant celui de la guerre ou de la défaite que l'avènement des ennemis de l'intérieur, qui ont supprimé toute liberté pour l'ensemble des Français et qui ont, en outre, exclu les Juifs comme lui du nombre des «vrais Français», lui qui se sentait, selon ses termes, «Français jusqu'à la moelle des os ${ }^{28}$. Il devait dire plus tard la brutalité de cette révélation:

Soudain me fut révélé, brutalement, que pour être classé Français, bon Français, il ne suffisait pas de l'être, pleinement, totalement, comme je l'étais, l'avais été toute ma vie; il fallait en plus être reconnu pour tel non seulement par l'ennemi tout-puissant vainqueur, mais par mes propres concitoyens ${ }^{29}$.

Or, l'exclusion de citoyens de toujours avait été aussi l'un des caractères majeurs des révolutions oligarchiques à Athènes. D'une manière qui peut surprendre, ce n'est pas contre les nazis, pourtant plus redoutables que Sparte, qu'il dirige sa colère, mais contre ses concitoyens « oligarques» qui ne sont mus que par la haine.

\section{JUGER, CLASSER, LIRE LES OLIGARQUES}

Dans ces conditions, ce n'est pas chose facile que de juger et de classer Les Oligarques, non plus que de définir son lectorat.

\section{Juger Les Oligarques}

a. Si l'on soumet Les Oligarques à une critique historique, on peut assurément contester la thèse de Jules Isaac selon laquelle les oligarques constituaient un groupe constant plus ou moins défini, depuis longtemps à l'affût d'une défaite pour s'imposer grâce à la collaboration avec l'ennemi: le parcours individuel des oligarques athéniens connus montre qu'ils ne l'ont pas toujours été - songeons à des acteurs aussi essentiels que Théramène, Alcibiade, Phrynichos ou Pisandre. La part de l'opportunisme et des fluctuations semble avoir été importante et les Athéniens eux-mêmes ne manquaient pas de le souligner à l'occasion dans leurs discours (il est vrai, pour appuyer les dénégations d'un accusé). Dans un plaidoyer de Lysias, le plaidant énonce cette généralité que l'on n'est pas oligarque ou démocrate par nature, mais en fonction de son intérêt personnel, qui varie suivant les circonstances ${ }^{30}$. Quant aux hétairies, elles restent trop fantomatiques dans nos sources et

(26) p. 8: «Plutôt que dans l'espace, étant de goûts sédentaires, il a choisi de s'enfuir dans le temps».

(27) «Survol. En guise d'introduction», Cahiers du Sud 376, 1964, p. 217-233, part. p. 226.

(28) Cité par Kaspi (supra n.16), p. 142.

(29) «Survol. En guise d'introduction» (supra n. 27), p. 225. Le pire était à venir, puisque, comme on l'a dit (supra n. 22), Jules Isaac devait voir disparaître, arrêtés par la Gestapo, sa femme, sa fille et son fils, avant d'apprendre après la guerre que les deux premières étaient mortes à Auschwitz.

(30) Lysias, Pour un citoyen accusé de menées contre la démocratie (25), 8-11. Voir aussi Isocrate, Sur la Paix (8), 133. 
elles étaient concrètement astreintes à une discrétion trop grande pour permettre une comparaison avec les tenants, bien voyants dans les années 1930, de l'extrême droite française. L'idée même de "parti oligarchique», qui revient plusieurs fois sous la plume de Jules Isaac, ne peut être retenue après examen des sources ${ }^{31}$.

Cette thèse traduit une vision que l'on trouvait déjà douze ans plus tôt dans le Malet-Isaac de $1930^{32}$ :

Pendant la guerre du Péloponnèse, les aristocrates d'Athènes n'avaient cessé de conspirer pour renverser le gouvernement démocratique. Dans les malheurs de leur patrie, ils ne virent qu'une occasion de ressaisir le pouvoir. Avec l'aide de Lysandre, ils formèrent le gouvernement des Trente.

L'auteur des Oligarques a même affiné le tableau, car il distingue désormais les oligarques comme une minorité parmi les aristocrates, la majorité étant à ses yeux inoffensive (p. 18-19). Néanmoins, quand on entre dans l'illustration de cette thèse étape par étape ${ }^{33}$, on constate un certain nombre d'inflexions et distorsions qui, cette fois, paraissent imputables à l'auteur de ce livre précis. Par exemple, si l'on suit Thucydide, l'interprétation des mutilations d'hermès comme un complot oligarchique n'était en fait qu'une rumeur peu crédible ${ }^{34}$. De même, le désastre de Sicile entraîna la consternation générale, mais aussi la détermination, tout aussi unanime, à poursuivre la guerre - sans qu'il soit fait état d'une exception oligarchique, que Jules Isaac invente pour les besoins du parallèle ${ }^{35}$. Rien ne donne à croire non plus à l'organisation de complots au sein des hétairies dès cette date. La désignation même de dix probouloi dès 413 n'a pas été conçue comme une concession à l'oligarchie ${ }^{36}$.

L'interprétation du procès des Arginuses comme un méfait proprement oligarchique peut prêter à discussion. Tel historien moderne a vu là une invention par laquelle Isaac avait voulu renforcer le parallélisme sur lequel repose tout son livre ${ }^{37}$. Il se pourrait que la réalité soit plus

(31) On pourra se reporter à l'étude prosopographique des acteurs du régime des Trente par György Németh, Kritias und die Dreißig Tyrannen. Untersuchungen zur Politik und Prosopographie der Führungselite in Athen 404/403 v. Chr., Stuttgart, 2006. Sur le caractère fluctuant de l'étiquette d'oligarque et sur l'absence d'un parti oligarchique au sens propre, cf. Martin Ostwald, "Oligarchy and Oligarchs in Ancient Greece», dans P. Flensted-Jensen, Th. Heine Nielsen et L. Rubinstein (éd.), Polis and Politics. Studies in Ancient Greek History, Copenhague, 2000, p. 385-396.

(32) Albert MaLet et Jules IsaAC, L’Orient et la Grèce, rédigé conformément aux programmes du 3 juin 1925. Classe de sixième, Paris, 1930, p. 345. Le chapitre en question a été rédigé en collaboration avec Gaston Dez (p. VI). Gustave Glotz fait partie des historiens de la Grèce remerciés pour leurs éclaircissements.

(33) Voir supra I.1.

(34) Cette distorsion est relevée par Curty (supra n. 1), p. 175. Cf. Thucydide, VI, 53 et 60. Le théâtre d'Aristophane témoigne dans le même sens. Cf. Dominique Lenfant, «Rois et tyrans dans le théâtre d'Aristophane», Ktèma 22, 1997, p. 185-200, aux p. 196-199. Cependant, il est peut-être déplacé de voir là une distorsion due à Isaac lui-même, car, comme le note Canfora dans sa préface à l'édition italienne (p. 23), l'interprétation des affaires de 415 comme complot oligarchique avait été soutenue par Henri Weil en 1900.

Curty (supra n. 1, p. 175) note à juste titre que Jules Isaac ne dit mot des arrestations arbitraires qui suivirent (cf. Thucydide VI,53,2). Il suppose qu'elles ont laissé «des traces profondes chez les Athéniens, la plupart issus des milieux aristocratiques, qui ont risqué d'être condamnés à mort», mais il se réfère sur ce dernier point à Lysias, Pour un citoyen accusé de menées contre la démocratie (25), 26, qui porte en fait sur les lendemains de la révolution des Quatre-Cents ( $\$ 25$ :

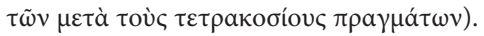

(35) J. IsaAc (p. 57-58), après avoir cité Thucydide, VIII, 1, sur l'accablement général qui frappe alors la cité, n’hésite pas à contredire ouvertement l'historien tout en admettant sa propre audace: «Moins discret que lui (par franche partialité), oserai-je affirmer, m’appuyant sur le témoignage des faits, que, dans la ville en deuil, la consternation n'était pas générale. Nul doute qu'elle n'était pas le sentiment dominant dans les hétairies oligarchiques» - une interprétation qui n'est qu'une simple supposition.

(36) Cf. Simon Hornblower, A Commentary on Thucydides. Volume III, Oxford, 2008, p. 752. C'est la contribution de ces dix «commissaires» à l'élaboration de la constitution oligarchique de 411 deux ans plus tard (Aristote, Constitution des Athéniens, 29,2) qui accentue rétrospectivement cette coloration.

(37) Curty (supra n. 1), p. 178: «Pour que joue le parallélisme entre les deux situations, il interprète le procès des stratèges vainqueurs aux Îles Arginuses (406) comme une action des oligarques et non, selon l'habitude, comme un fait imputable à la démocratie extrême.» «Pour Isaac, tout s'explique par le recours systématique au complot oligarchique.» 
complexe. D'un côté, pour nombre d'historiens modernes, l'événement tel que le décrit Xénophon est l'illustration des «horreurs» dont la démocratie est capable ${ }^{38}$ : si les généraux sont condamnés à mort sans bénéficier d'un procès légal, c'est parce que l'Assemblée se laisse convaincre qu'il serait scandaleux de ne pas laisser le peuple faire ce qu'il veut ${ }^{39}$. De plus, les orateurs influents en cette affaire ne peuvent être identifiés en toute sûreté comme des oligarques ${ }^{40}$, ce qui fait de l'affirmation de Jules Isaac une hypothèse fragile.

D'un autre côté, on peut soupçonner le récit de Xénophon d'être biaisé par le parti pris antidémocratique de son auteur. Et, sans entrer dans le cœur d'une affaire qu'il n'y a pas lieu d'analyser ici dans le détail, il faut se rappeler la possibilité d'une action masquée qui aurait laissé peu de traces dans nos maigres sources, fort peu diversifiées. Ce qui importe à notre propos est qu'Isaac, loin de l'avoir conçue lui-même pour les besoins de sa thèse, emprunte en fait l'idée d'un complot oligarchique à un historien de l'Antiquité, Paul Cloché, auteur d'une analyse très poussée de l'affaire, parue en 1919 et à laquelle Isaac se réfère explicitement ${ }^{41}$. Du reste, l’idée du procès comme manipulation mise en scène par l'oligarque Théramène a été défendue récemment encore, non sans de bons arguments, par Cinzia Bearzot ${ }^{42}$. C'est là une autre hypothèse qui ne peut déboucher sur une certitude absolue, mais qui fait de la position de Jules Isaac non pas une distorsion due à sa thèse, mais une grille de lecture adoptée par certains spécialistes. Tout juste pourrait-on lui reprocher d'en faire plus qu'une hypothèse ${ }^{43}$.

Enfin, pour revenir au thème de la défaite, il n'est pas sûr que le désastre de 405 ait été invoqué pour discréditer la démocratie en tant que telle - du moins n'en trouve-t-on aucune trace dans nos sources $^{44}$.

(38) Voir, entre autres exemples, Édouard WiLl, Le monde grec et l'Orient - Le Ve siècle (510-403), Paris, 1972 (19916), p. 387-388: «À leur retour à Athènes, les stratèges vainqueurs furent mis en accusation et, au terme d'assemblées tumultueuses où les haines personnelles et la démagogie le disputèrent à la piété la plus superstitieuse, ils furent collectivement condamnés à mort»; l'historien qualifie ensuite cette condamnation d' "acte de délire collectif", avant de conclure: "Que le peuple eût envoyé à la mort certains de ses meilleurs serviteurs, un Diomédon, un Thrasyle, voilà qui pouvait fournir des arguments aux oligarques.» En d'autres termes, l'événement est imputable au régime démocratique et, si les oligarques en ont tiré argument contre ce dernier, c'est sans y avoir joué le moindre rôle.

Mogens H. Hansen, La démocratie athénienne à l'époque de Démosthène, Paris, 1993, p. 65-66, affirme même que le procès «fut cité par les contemporains comme preuve que la démocratie d'assemblée était une mauvaise forme de gouvernement», mais les sources auxquelles il renvoie en note ne disent rien de tel.

(39) Xénophon, Helléniques, I, 7, 12. Cf. Edmond LÉvy, «L'art de la déformation historique dans Les Helléniques de Xénophon», dans H. Verdin, G. Schepens et E. De Keyser (éd.), Purposes of History. Studies in Greek Historiography from the 4th to the 2nd Centuries B.C., Louvain, 1990, p. 125-157.

(40) Callixène, l'orateur qui proposa le jugement en bloc des stratèges, est soupçonné par Isaac (p. 101) d'être un «oligarque masqué», du fait qu'il est ensuite passé à Décélie, où étaient alors les Spartiates. De fait, d’après Xénophon comme selon Diodore, peu après la condamnation des généraux, le peuple se repentit, accusa Callixène et les autres orateurs de l'avoir trompé et les fit emprisonner en attendant leur procès, ce qui les incita à s'évader (Helléniques, I, 7, 35) : c'est dans ces circonstances que Callixène serait passé à Décélie (d'après Diodore, XIII, 103,2). Toutefois, la seule chose que l'on sache sur Callixène à la suite de sa fuite est ce qu'en dit Xénophon (Helléniques, I, 7,35): il rentra à Athènes avec les exilés du Pirée - soit avec les démocrates en 403, et non avec les oligarques en 404 (ce qui paraît étrange s'il s'était bien réfugié auprès des Lacédémoniens, si bien que l'on peut s'interroger sur la compatibilité entre la version de Xénophon et celle de Diodore).

(41) Paul Cloché, "L’affaire des Arginuses (406 avant J.-C.)", Revue historique 130/1, 1919, p. 5-68 (sur l'idée de complot: p. 49-51). Isaac s'y réfère p. $101 \mathrm{n} .1$.

(42) Cinzia Bearzot, Come si abbatte una democrazia. Tecniche di colpo di Stato nell'Atene antica, Bari, 2013, p. 89-108.

(43) À l'inverse, BeArzot (supra n. 42, p. 107) admet que son hypothèse n'est pas démontrable en toute rigueur.

(44) Hansen (supra n.38, p. 66) affirme le contraire, mais sans citer de source à l'appui: "Les conséquences constitutionnelles furent alors énoncées: la démocratie a fait faillite, disait-on.»).

À en croire Lysias, Contre Ératosthène (12), 74-75, ou Aristote, Constitution des Athéniens, 34,3, ce fut le seul appui du Spartiate Lysandre qui fit pencher la balance du côté des oligarques, c'est-à-dire l'intimidation et la terreur, et non l'idée que la défaite était imputable au régime démocratique et à sa faiblesse. Ce qui est, en revanche, incontestable, c'est que les oligarques entendirent profiter de l'affaiblissement dû à la défaite, qui leur parut une véritable aubaine (par exemple, Lysias, 
De tels infléchissements servent le propos de l'auteur. Ainsi, en imputant aux oligarques la tournure prise par le procès des Arginuses, il croit pouvoir mieux montrer la présence constante et maléfique des oligarques et sans doute veut-il aussi laver la démocratie athénienne d'un comportement qui fait tache dans le tableau idéal qu'il aime à en donner ${ }^{45}$.

b. Le filtre de l'analogie n'est pas sans conséquence. Certes, bien des ressemblances sont frappantes et judicieusement mises en lumière par l'auteur. Comme l'a noté Pascal Ory, «ce n'est pas parce que l'histoire ne se répète pas que les êtres qui la font - qui la sont - ne sont pas mus par des forces étonnamment semblables ${ }^{46}$ et, de ce point de vue, on veut bien croire que tout n'a pas changé entre Athènes et Vichy. Néanmoins, Jules Isaac ne se contente pas d'exploiter les analogies: il lui arrive de les construire, consciemment ou non, et d'interpréter l'histoire athénienne suivant le modèle français, en attribuant, par exemple, aux oligarques athéniens les arrière-pensées des fascistes français. Il tend aussi à ne retenir des années 415-404 que ce qui lui paraît correspondre aux événements des années 1930-1940.

c. Le manque de renvois aux sources se fait parfois sentir, surtout quand on a des doutes sur l'analogie sous-jacente avec un phénomène du xx siècle qui pourrait être en fait la véritable origine de l'affirmation - par exemple, sur l'idée que la défaite aurait permis de jeter le discrédit sur la démocratie. Il est vrai que la discrétion des sources est une difficulté de tout sujet lié à des agissements secrets. Leur silence est en l'occurrence propice aux hypothèses, dont toutes ne sont pas invraisemblables. Il faut ainsi avouer que nous connaissons mal les activités souterraines des oligarques, qui ne sont sans doute pas que fantasme. Quand ils font appel à Lysandre pour qu'il menace les citoyens qui ne voteraient pas le décret de Dracontidès remettant les pouvoirs aux Trente $^{47}$, il n'est pas insensé de supposer avec Jules Isaac qu'un accord secret a permis de préparer le coup d'État (p. 124-126). Néanmoins, un historien pourrait reprocher à l'auteur d'affirmer parfois avec force ce qui ne peut qu'être hypothèse. Du reste, il signale plus d'une fois le silence des sources $^{48}$, voire le fait qu'il les contredise par parti pris ${ }^{49}$.

d. Quant aux jugements de valeur émis par l'auteur, certains critiques se sont montrés sévères $^{50}$, quand d'autres ont considéré que l'on pouvait aisément les disjoindre d'un récit par ailleurs honnête ${ }^{51}$. Il est vrai que ce ne sont pas les jugements désapprobateurs qui gênent le lecteur. Mais le filtre moral a d'autres conséquences. Par exemple, la définition même des oligarques en termes psychologiques et moraux, comme simplement animés par la haine et la cupidité, omet de présenter leur théorie de la bonne distribution des pouvoirs - à ceux qui contribuent au bien public

Contre Ératosthène (12), 44; Contre Agoratos, 6). Que, par la suite, les démocrates aient désigné comme un malheur tout à la fois la défaite et ses conséquences, perte de la flotte et des Longs Murs, mais aussi renversement du régime (E. LÉvY, Athènes devant la défaite de 404. Histoire d'une crise idéologique, Paris, 1976, p. 40-41), voilà qui est encore une autre affaire.

De même, le caractère purement idéologique de la guerre du Péloponnèse (entre adversaires et partisans de la démocratie) affirmé p. 11 est discutable et même contraire à ce qu'en dit Thucydide, qui est pourtant la source revendiquée par Isaac.

(45) Voir sa présentation exaltée de la démocratie athénienne et du principe de liberté qui l'anime (p. 12-17). Il n'est pas exclu que, pour cette même raison, il néglige de rechercher les sources de mécontentement que pouvait générer la démocratie, à Athènes comme en France: Chandezon (supra n. 1, p. 102-103) souligne que la corruption (présumée) des démagogues athéniens était un point de convergence possible avec les «affaires » des années trente et il suppose qu'Isaac en a gommé l'existence pour ne pas justifier les réactions, en particulier chez ses contemporains de la III République.

(46) ORY (supra n. 16), p. XI.

(47) Lysias, Contre Ératosthène, 72-75.

(48) C'est le cas, par exemple, pour l'action souterraine des hétairies entre 413 et 411 (p. 60).

(49) Voir supra, n. 35 à propos de la réaction athénienne au désastre de Sicile.

(50) Curty lui reproche de présupposer que la démocratie est le bien et l'oligarchie le mal (supra n. 1, p. 179).

(51) Ory (supra n.16), p. IX, note que les jugements de valeur d'Isaac «sont si clairement affichés qu'ils sont disjoignables du récit honnête des faits, à quoi se ramène son propos». 
par leur personne et leur argent ${ }^{52}$. Pour dire le vrai, c'est là un choix parfaitement conscient et faire la leçon à Jules Isaac en comparant son récit à un manuel d'aujourd'hui - comme cela s'est fait ${ }^{53}$ pourrait être hors de propos.

\section{Classer Les Oligarques}

Jules Isaac a été le premier à prendre la casquette de l'historien pour anticiper les critiques de ses collègues. Au cœur de son récit, il s’interrompt et imagine un lecteur qui lui reproche «cette partialité qui s'étale sans vergogne jusque sur la couverture de l'ouvrage, cette impudente liberté d'interprétation qui donne au passé la couleur du présent, ce langage de pamphlétaire plus que d'historien» (p. 92). On le voit: partialité, lecture analogique, voire anachronique, jugements de valeur, tout y est pleinement assumé. L'auteur n'est ni un naïf ni un imposteur ${ }^{54}$. Il affiche la couleur dès le sous-titre de l'ouvrage: Essai d'histoire partiale. On peut certes y voir une provocation, mais c'est aussi une marque de modestie («essai») et d'honnêteté intellectuelle («partiale»), un avertissement au lecteur de la part d'un historien qui s'est toujours distingué par son souci de vérité et de rigueur ${ }^{55}$. En d'autres termes, il faut lui épargner un faux procès: l'auteur ne voyait pas luimême ce livre comme destiné à l'enseignement de l'histoire, et c'est peut-être la raison pour laquelle il ne l'a jamais publié sous son nom.

Comment faut-il donc classer le livre? Il est plus facile de dire ce qu'il n'est pas: ni un roman historique ni de l'histoire antique ${ }^{56}$ ni de l'histoire du temps présent ni de l'histoire comparée puisqu'il ne met en valeur que les ressemblances sans effectuer de comparaison ni jamais préciser les différences ${ }^{57}$. Ce n'est pas un ouvrage pour spécialistes, pas un ouvrage pour historiens soucieux d'impartialité, pas un ouvrage universitaire ni un manuel. Il tient de l'essai bien documenté, de l'histoire allusive ${ }^{58}$ et du pamphlet, ce qui pose la question de son public.

(52) Thucydide, VIII, 65, 3; Aristote, Constitution des Athéniens, 29, 5.

(53) C'est la démarche adoptée par CurTy (supra n.1), lorsqu'il compare le livre d'Isaac au manuel général écrit à l'intention des étudiants par Edmond Lévy, La Grèce au ve siècle de Clisthène à Socrate, Paris, 1995 (non sans d'étranges considérations sur le fait que ce dernier «est, comme Jules Isaac, un historien d'origine juive. Cependant, vivant à une époque où l'antisémitisme est officiellement rejeté, il n'en subit pas, comme Isaac, les pénibles conséquences et peut apparaitre comme plus modéré», p. 172).

(54) CuRty (supra n. 1, p. 180) donne à penser le contraire quand il affirme qu'Isaac «ne s'embarrasse pas de scrupules excessifs».

(55) Cf. Kaspi (supra n. 16), p. 138; Jacques Madaule, dans Jules Isaac. Actes du colloque. Catalogue de l'exposition organisée par l'Université de Haute-Bretagne (Rennes II), 28 novembre-10 décembre 1977, Paris, Hachette, 1979, p. 5: «S'il ne fallait user que d'un seul mot pour caractériser Jules Isaac, j'userais volontiers de celui de rigueur. » Rappelons d'ailleurs qu'au début de la Seconde Guerre mondiale, Isaac avait défendu avec force le principe d'un enseignement de l'histoire qui ne subirait pas l'influence de la guerre: "Il n'y a pas d'enseignement historique pour temps de guerre. Aujourd'hui comme hier, l'enseignement historique se doit de rester fidèle à ce haut idéal de droiture et de probité scrupuleuse qui le maintient dans la plus saine tradition française et le préserve de toute contagion d'idolâtrie» (extrait de discours cité par KASPI [supra n. 16], p. 138).

(56) Cela n'exclut pas que, comme le dit Chandezon (supra n. 1, p. 97), Jules Isaac ait néanmoins eu aussi l'ambition d'amener «les historiens de la Grèce antique à réfléchir sur les événements qui avaient conduit au régime oligarchique de 404-403».

(57) Parmi les différences importantes figure le fait qu'en 404 l'intervention de Sparte fut déterminante pour pousser les Athéniens à renoncer à leur régime démocratique, alors que les Français le firent sans intervention aucune de l'Allemagne, leurs députés ayant voté à l'Assemblée la remise à Pétain des pleins pouvoirs constituants. Le silence sur les différences fait que j'hésiterais peut-être à parler d'histoire comparée comme l'a fait Christophe CHANDEzon (supra n. 1). Les ressemblances avec Vichy ne sont elles-mêmes que suggérées et implicites, la comparaison n'intervenant, on l'a dit, que dans la formule conclusive.

(58) J'emprunte l'expression à Canfora dans sa préface à la traduction italienne (supra n. 1), p. 32. 


\section{Lire Les Oligarques}

Le lectorat des Oligarques n'est pas aisé à définir. Si l'écrit s'apparente à un pamphlet, que dire des cibles qu'il visait? S'en prendre à des hommes d'il y a vingt-trois siècles n'aurait pas grand sens. Et que penser d'une attaque aussi codée contre des contemporains? Du reste, le livre ne fut publié - au regret de l'auteur - qu'en avril 1945: le Régime de Vichy était mort depuis huit mois ${ }^{59}$.

On est frappé de l'accumulation des handicaps dont put souffrir sa promotion. En premier lieu, le titre n'était pas fait pour attirer les foules ${ }^{60}$. L'absence de référence à l'Antiquité est parfaitement délibérée, puisqu'il s'agit de suggérer une analogie avec les collaborateurs et militants d'extrême droite des années 1930-1940. C'est aussi une marque d'honnêteté: l'auteur ne prétendait pas avoir écrit de la «pure» histoire grecque.

En second lieu, la publication de 1945 s'est faite sous le seul pseudonyme de Junius - ce qui était une allusion savante que bien peu de gens pouvaient alors décrypter ${ }^{61}$. On peut supposer que sous le nom du très connu Jules Isaac le livre se serait mieux diffusé ${ }^{62}$. Sans doute était-ce là le pseudonyme sous lequel l'auteur avait espéré publier son livre de résistance à l'époque de Vichy. Peut-être aussi a-t-il eu scrupule à mêler son nom à une œuvre à caractère pamphlétaire, qui se démarquait en cela de son œuvre antérieure de pédagogue, comme de son travail d'investigation en cours sur les racines de l'antisémitisme chrétien.

Toujours est-il que cette absence de concession contribua sans doute à l'échec commercial du livre, qui de clandestin devint confidentiel. La raison fondamentale était cependant dans le rapport du livre avec une actualité qui avait changé ${ }^{63}$. Ce n'est qu'en 1989, bien après la mort de l'auteur ${ }^{64}$, que le livre fut republié, cette fois sous son nom, avec une préface du contemporaniste Pascal Ory. Il n'a guère retenu l'attention que de deux types de spécialistes: soit des contemporanistes intéressés par la personnalité de Jules Isaac, soit des antiquisants francophones intéressés par ce curieux récit hybride. Il n'a été traduit que tout récemment en italien et ne paraît pas l'avoir été dans d'autres langues ${ }^{65}$.

C'est pourtant un petit chef-d'œuvre littéraire, un récit d'histoire vivant et plein de verve, écrit par un grand esprit, en même temps qu'une mise en parallèle stimulante, un pamphlet savant, le cri (maîtrisé) d'un homme meurtri, un hymne à la liberté, un livre pour les happy few qui peuvent en savourer le nectar. Relégué jusqu'ici au rang des curiosités, il mériterait d'intéresser davantage et d'être traduit plus largement pour mieux franchir les frontières.

\section{Dominique Lenfant Université de Strasbourg, UMR 7044 Archimède}

(59) Rappelons qu'il fut publié aux éditions de Minuit, nées dans la clandestinité sous l'Occupation allemande et dont le premier titre avait été, en 1942, Le Silence de la Mer de Vercors. L'auteur avait voulu le publier plus tôt, mais en vain (il devait écrire dans «Survol» (supra n. 27), p. 226: «Je n'avais le choix qu’entre une publication clandestine en France, ou libre à l'étranger; à cette date, 1942, je n'obtins ni l'une ni l'autre»).

(60) KASPI (supra n. 16), p. 156. Aujourd'hui, il prêterait sans doute à confusion, puisque les oligarques désignent les nouveaux riches de la Russie post-soviétique (ainsi, dans le livre de Christine Ockrent intitulé Les Oligarques. Le système Poutine, Paris, 2014), ou les grandes fortunes qui dominent certains États.

(61) Comme l'explique Chandezon (supra n. 1, p. 97), le nom de Junius renvoyait à une publication londonienne du $\mathrm{XVIII}^{\mathrm{e}}$ siècle, due à un polémiste anonyme, qui dénonçait le gouvernement tyrannique de George III, les Letters of Junius, parues vers 1770. KASPI (supra n. 16, p. 156) souligne le caractère codé de l'allusion.

(62) L'Année philologique de 1945-1946, p. 341, cite Junius en décryptant le pseudonyme par l'indication "[J. Isaac]", mais elle a été publiée en 1948 et ne s'adressait qu'à un public de spécialistes. Il en va de même des comptes rendus d'Aymard et de Cloché parus en 1947 et 1948 (supra n. 1).

(63) Sur les causes de cet échec commercial, nous reprenons les analyses de KASPI (supra n. 16), p. 156-159.

(64) Jules Isaac est mort en 1963.

(65) Au contraire, le livre de Jules Isaac sur les racines chrétiennes de l'antisémitisme, (J. IsAAC, Jésus et Israël, composé à partir de 1943 et paru en 1948) fut traduit en anglais en 1971. Il avait contribué à inspirer le texte de Vatican II, par lequel l'Église catholique affirmait renoncer à tout discours antijudaïque. 